\title{
Preoperative short-course radiotherapy followed by consolidation chemotherapy for treatment with locally advanced rectal cancer: a meta-analysis
}

Haoyan $\mathrm{Wu}^{1,2 \dagger}$, Chuanwen Fan ${ }^{1,2,4,5,6,7 \dagger}$, Chao Fang ${ }^{1,2 \dagger}$, Libin Huang ${ }^{1,2}$, Yuan $\mathrm{Li}^{1,3}$ and Zongguang Zhou ${ }^{1,2^{*}}$ (1)

\begin{abstract}
Background: The addition of consolidation chemotherapy to preoperative short-course radiotherapy during the prolonged interval between the completion of radiation and surgery in locally advanced rectal cancer (LARC) could enhance pathologic response and might act on potential micrometastasis. We performed this meta-analysis to evaluate whether short-course radiotherapy followed by consolidation chemotherapy (SCRT/CCT) could be a neoadjuvant treatment option compared with conventional long-course chemoradiotherapy (LCCRT).
\end{abstract}

Methods: We searched the PubMed, EMBASE, MEDLINE, and Cochrane Library databases. The primary endpoints were pathological outcomes, and the secondary endpoints included survival rate, sphincter preservation rate, R0 resection rate and toxicity. RevMan 5.3 was used to calculate pooled risk ratio (RRs) and 95\% confidence intervals (Cls).

Results: A total of seven eligible studies and 1865 participants were included in this meta-analysis. Compared with the LCCRT, SCRT/CCT increased pathologic complete response $(\mathrm{pCR})$ rate $[R R=1.74,95 \% \mathrm{Cl}(1.41,2.15), \mathrm{P}<0.01]$ and led to a lower proportion of patients with adjuvant pathologic tumor stage 3-4 (ypT3-4) disease $[R R=0.88,95 \% \mathrm{Cl}$ $(0.80,0.97), P=0.01]$ or lymph node positive $(y p N+)$ disease $[R R=0.83,95 \% \mathrm{Cl}(0.71,0.98), P=0.02]$. In addition, the disease-free survival (DFS) was better in SCRT/CCT group $[R R=1.10,95 \% \mathrm{Cl}(1.02,1.18), \mathrm{P}=0.01]$, while overall survival rate and toxicity and surgical procedures were similar between two groups.

Conclusion: Based on better pathological outcomes and DFS in SCRT/CCT group, we recommended preoperative short-course radiotherapy followed by consolidation chemotherapy as the optional neoadjuvant treatment for LARC.

Keywords: Rectal cancer, Short-course radiotherapy, Consolidation chemotherapy, Meta-analysis

\section{Background}

There are two general approaches to preoperative neoadjuvant treatment for locally advanced rectal cancer (LARC). Conventionally chemoradiotherapy (CRT),

\footnotetext{
*Correspondence: zhou767@163.com

${ }^{\dagger}$ Haoyan Wu, Chuanwen Fan and Chao Fang contributed equally to this work

${ }^{1}$ Laboratory of Digestive Surgery, State Key Laboratory of Biotherapy and Cancer Center, Sichuan University, Chengdu 610041, China

Full list of author information is available at the end of the article
}

consisting of long-course radiotherapy with concomitant fluoropyrimidine chemotherapy, is the current standard treatment for TNM stage II and III rectal cancer in the United States and southern Europe, whereas short-course radiotherapy (SCRT) with immediate surgery is more commonly applied in the north Europe [1-3]. Both preoperative neoadjuvant treatments, with similar safety and efficacy, have decreased local recurrence and improved survival rate [4-6]. The distant disease recurrence, 
however, has not decreased accordingly and remained a substantial problem.

SCRT with immediate surgery, which is advised for those with intermediate risk rectal cancer or contraindications to long-course radiation, is inferior to conventional CRT in terms of pathologic complete response (pCR) rate and tumor downstaging. Desired pathological outcomes occur when surgery is delayed after SCRT, as found in the Stockholm III trial and a systematic review in 2014 [3, 7, 8], which both addressed the optimal interval between the completion of radiotherapy and resection of tumor. The interval could be prolonged appropriately, creating an opportunity to deliver systemic chemotherapy preoperatively, which, to some extent, might act on obscure micrometastases and thereby reduce distant metastasis [9]. Some centers have administered postoperative adjuvant chemotherapy with the intention of reducing distant failure, but the effect and compliance were far from satisfactory [10]. However, the upfront systemic chemotherapy, which is delivered in the waiting period between SCRT and resection, is well-tolerated, as reported by several trials and the cooperation regimen of consolidation chemotherapy and delayed surgery after SCRT had high neoadjuvant therapy completion rate and tumor downstaging [11-14].

Based on these findings, it is concluded that such a combination of the prolonged waiting period between the completion of SCRT and resection and delivering consolidation chemotherapy during the interval might be superior to conventional long-course chemoradiation. Herein, we report a meta-analysis of all those published studies adopting the short-course radiotherapy followed by consolidation chemotherapy (SCRT/CCT) for LARC with the aim of comparing pathological outcomes and survival rates to those of conventional long-course chemoradiotherapy (LCCRT).

\section{Material and methods Inclusion criteria}

According to the PICOS principles, we defined the following inclusion criteria: (1) Participants (P): studies involving patients with nonmetastatic rectal cancer confirmed by biopsy and receiving neoadjuvant treatment. (2) Interventions (I) and comparisons (C): studies comparing SCRT/CCT with LCCRT as neoadjuvant treatment in LARC. The SCRT/CCT regimen was 25 Gy in five fractions, regardless the use of concurrent chemotherapy, followed by several cycles of consolidation chemotherapy before surgery; the LCCRT regimen was long-course radiotherapy with concomitant chemotherapy followed by surgery. (3) Outcomes (O): studies evaluating following outcomes: pCR rate, adjuvant therapy pathologic stage(ypTNM), local recurrence (LR), distant metastasis (DM), overall survival rate (OS), disease free survival rate (DFS), sphincter preservation rate, radical (R0) resection rate, postoperative complications, downstaging rate, acute toxicity and late complications. (4) Study design (S): prospective and retrospective studies.

We excluded the following publications: (1) studies involving patients with synchronous metastases or serious cardiopulmonary diseases or other severe basic diseases; (2) short-course radiotherapy without consolidation chemotherapy before surgery; (3) studies were not controlled trials, for example, single arm study, case series or case report; (4) studies lacking complete important information for extracting the required data; and (5) non-original studies, such as letters, reviews, and expert opinions.

\section{Literature search}

We systematically searched the PubMed, MEDLINE, EMBASE, and the Cochrane Controlled Trials Register by using terms of "short-course radiotherapy", "chemotherapy", "long-course chemoradiotherapy", Medical Subject Heading (MeSH) terms "rectal Neoplasms" and its individual corresponding free terms with combination of Boolean operators (AND, OR, NOT). There was no language restriction. The last search was updated on 21 July, 2021. In addition, we reviewed references in the retrieved articles to search for additional relevant studies.

\section{Assessing risk of bias of included studies}

The quality of randomized controlled trials (RCTs) was assessed using the Cochrane Collaboration's risk for bias assessment tool [15], which evaluated the selection bias, performance bias, detection bias, attrition bias, and reporting bias. The quality of cohort studies was measured by a score system assessed in accordance with the Newcastle-Ottawa criteria [16]. The total scores ranged from 0 (worst) to 9(best) for cohort studies, with a score of at least 6 indicating high quality. Each criterion was assessed as low risk for bias, high risk for bias, or uncertain risk for bias.

\section{Data extract}

The following information were extracted from each selected paper if available: first author, year of publication, number of patients, type of study, follow-up time, intervention and comparison, OS, DFS, LC, DM, sphincter preservation rate, $\mathrm{R} 0$ resection rate, postoperative complications, pCR rate, downstaging rate, ypTNM stage, acute toxicity and late complications.

\section{Data analysis}

All statistical analyses were performed using RevMan 5.3 software. Count data using risk ratio (RR), and $95 \%$ 
confidence intervals (CI) was calculated. Heterogeneity was assumed by using the $\mathrm{I}^{2}$ method with the $\chi^{2}$ test to calculate $\mathrm{P}$ values. If heterogeneity was not present $\left(\mathrm{P}>0.10, \mathrm{I}^{2}<50 \%\right)$, a fixed-effect model was adopted for analysis, otherwise, a random-effect will be employed.

\section{Results}

\section{Study selection}

A total of 969 relevant articles were searched, and 364 duplicates were removed. After reviewing the titles and abstracts, 593 of the studies were excluded due to irrelevant. Next, 12 potential eligible full-text articles were further evaluated. We excluded another 5 full-text articles, including 3 articles for not meeting the criteria for SCRT/ CCT and 2 articles that were the same study described at different time point. Finally, we included seven studies in the meta-analyses (Fig. 1). Four of these studies were RCTs [9, 17-19], and the other three were non-RCTs [20-22].

All of the involved RCTs mentioned that patients were informed of their treatment plan at allocation. However, this limitation was unlikely to affect the results of quality assessment (Additional file 1: Fig. 1 and Additional file 1: Fig. 2, which demonstrated risk of bias graph and risk of bias summary). The three non-RCTs were all cohort studies including two prospective study [20,22] and one retrospective study [21]. All three cohort studies scored at least 6 based on the Newcastle-Ottawa criteria (Additional file 1: Table 1).

A total of 1865 patients with locally advanced rectal cancer were assigned to the SCRT/CRT group $(n=928)$ or LCCRT group $(n=937)$. The characteristics of studies and patients, shown in Table 1 and Additional file 1: Table 2, were similar between the two treatment groups.

\section{Primary endpoint: pathological outcomes}

The pathological outcomes consisted of the pCR rate, downstaging rate and ypTNM stage. All seven trials were available for comparative analysis of $\mathrm{pCR}$ rate. As shown in Fig. 2, the pCR rate was obviously higher in the SCRT/ CCT group $[R R=1.74,95 \%$ CI $(1.41,2.15), \mathrm{P}<0.01$; $\mathrm{I}^{2}=0 \%$, fixed-effect model]. With respect to downstaging rate, available in five studies involving 434 patients, difference was not significant among recipients of two arms $\left[R R=1.19,95 \%\right.$ CI $(0.86,1.66), \mathrm{P}=0.30 ; \mathrm{I}^{2}=78 \%$, random-effect model; Additional file 1: Fig. 3]. Five trials, with a total of 1581 patients, reported ypTNM stage and pooled results suggested that the SCRT/CCT group had a lower proportion of ypT3-4 patients $[\mathrm{RR}=0.88,95 \% \mathrm{CI}$ (0.80, 0.97), $\mathrm{P}=0.01 ; \mathrm{I}^{2}=31 \%$, fixed-effect model; Additional file 1: Fig. 4] and $y \mathrm{pN}+$ patients $[\mathrm{RR}=0.83,95 \% \mathrm{CI}$ $(0.71,0.98), \mathrm{P}=0.02 ; \mathrm{I}^{2}=0 \%$, fixed-effect model; Additional file 1: Fig. 5].

\section{Secondary endpoints: survival rates, toxicity and surgical procedures}

The survival events in the involved trials were assessed, when available, at fixed time points (as provided in each study). The median follow-up duration ranged from 22.6 to 84 months.

Survival rates, including OS, DFS, LR and DM, were analyzed. No statistic difference in OS from four available studies was observed between the SCRT/CCT group and LCCRT group $[R R=1.03,95 \%$ CI $(0.97$, 1.08), $\mathrm{P}=0.36 ; \mathrm{I}^{2}=0 \%$, fixed-effect model; Fig. 3]. DFS, reported in five studies with a total of 1665 patients, was significantly better in the SCRT group $[R R=1.10$, $95 \%$ CI $(1.02,1.18), \quad \mathrm{P}=0.01 ; \mathrm{I}^{2}=0 \%$, fixed-effect model; Fig. 4].

LR and DM were reported in five studies. Incidence of LR did not significantly differ between the SCRT/ CCT cohort and LCCRT cohort $[R R=1.19,95 \% \mathrm{CI}$ $(0.95,1.50), \mathrm{P}=0.13 ; \mathrm{I}^{2}=0 \%$, fixed-effect model; Additional file 1: Fig. 6]. Despite higher incidence of DM in the SCRT/CCT group, however, the difference did not reach statistical significance $[R R=0.70,95 \%$ CI $(0.45$, 1.07), $\mathrm{P}=0.10 ; \mathrm{I}^{2}=68 \%$, random-effect model; Additional file 1: Fig. 7].

Toxicity, including acute toxicity, postoperative complications and late complications, was mentioned in all of the included studies. Acute toxicity, which was classified according to the Common Terminology Criteria for Adverse Events and postoperative complications were defined as complications that occurred within 30 days after resection. We assessed only grade three or higher adverse events based on the available data. There were no statistically significant differences between two arms $\left[R R=1.31,95 \%\right.$ CI $(0.92,1.86), P=0.13 ; I^{2}=66 \%$, random-effect model; Additional file 1: Fig. 8]. RR for post-operation complications and late complications were 1.12 and 1.18 , respectively [ $95 \%$ CI $(1.00,1.26)$, $\mathrm{P}=0.06 ; \mathrm{I}^{2}=0 \%$, fixed-effect model; Additional file 1 : Fig. 9] [ $95 \%$ CI $(1.00,1.40), P=0.05 ; I^{2}=29 \%$, fixedeffect model; Additional file 1: Fig. 10], which were close to borderline significance.

The surgical procedures included the $\mathrm{R} 0$ resection rate and sphincter preservation rate. $\mathrm{R} 0$ resection rate was comparable between the two neoadjuvant treatment groups $[R R=1.04,95 \% \mathrm{CI}(1.00,1.09), \mathrm{P}=0.08$; $\mathrm{I}^{2}=0 \%$, fixed-effect model, Additional file 1: Fig. 11], so was sphincter preservation rate $[R R=1.03,95 \% \mathrm{CI}$ $(0.93,1.14), \mathrm{P}=0.57 ; \mathrm{I}^{2}=0 \%$, fixed-effect model; Additional file 1: Fig. 12]. 


$$
\begin{aligned}
& \text { Records identified through } \\
& \text { database searching } \\
& (\mathrm{n}=964)
\end{aligned}
$$

\section{Additional records identified}

through other sources

$$
(\mathrm{n}=5)
$$

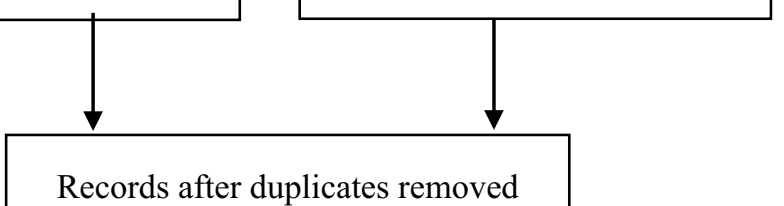

Records after duplicates removed

$$
(\mathrm{n}=605)
$$

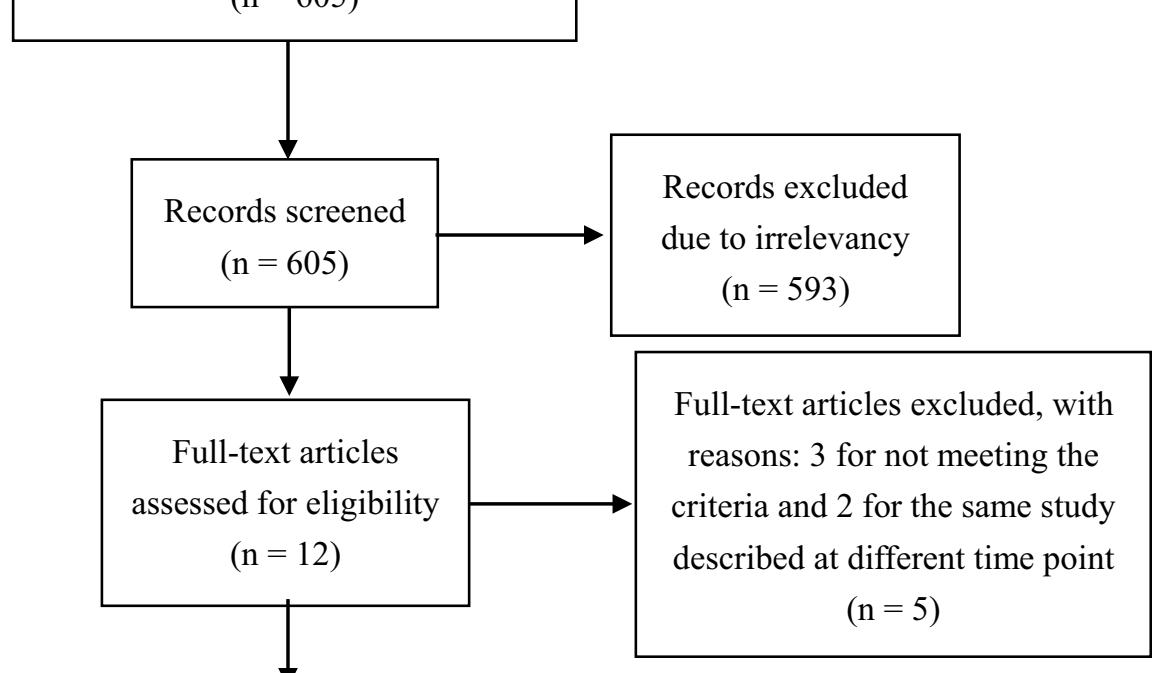

Studies included in

qualitative synthesis

$$
(\mathrm{n}=7)
$$

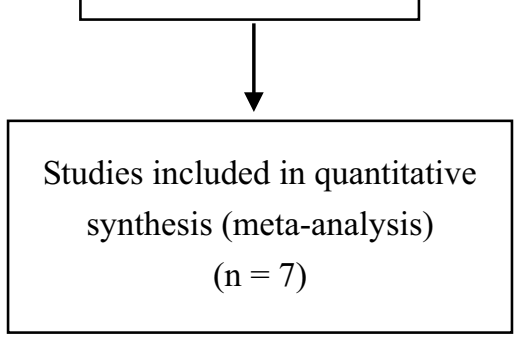

Fig. 1 PRISMA 2009 flow diagram

\section{Discussion}

The meta-analysis demonstrated that the SCRT/CCT group had better DFS and $\mathrm{pCR}$ and a lower proportion of ypT3-4 stage patients and ypN + patients than the LCCRT group. The OS and oncological outcomes in the SCRT/CCT group were similar to those in the conventional LCCRT group, as were the toxicity and surgical procedures.
An increased pCR rate and favorable adjuvant pathologic stage were observed in the SCRT group, which could be attributed to delayed surgery and the addition of chemotherapy. Nevertheless, such advantages did not translate to survival benefits or surgical procedures. The prolonged interval between radiotherapy and surgery resulting in pCR benefits has been verified by several randomized trials $[7,23]$. The addition of chemotherapy 


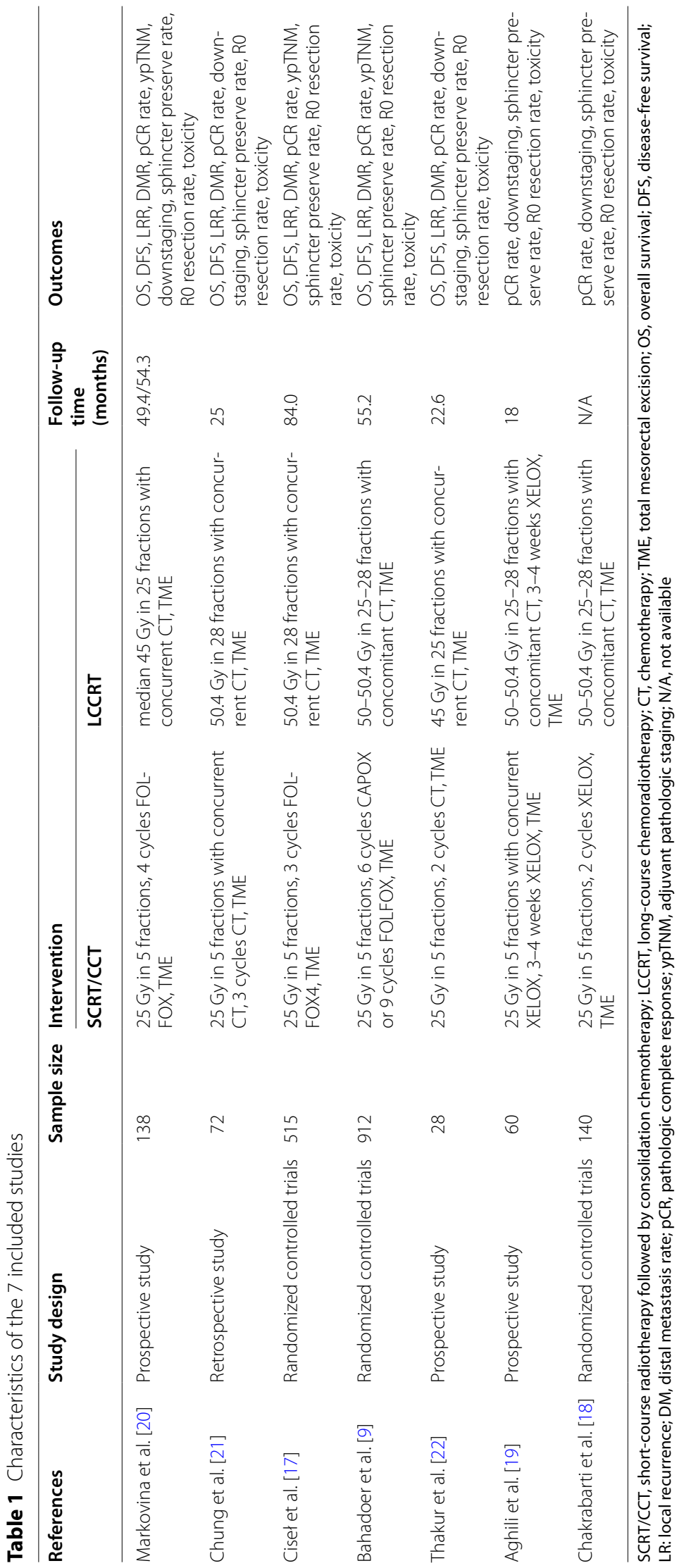




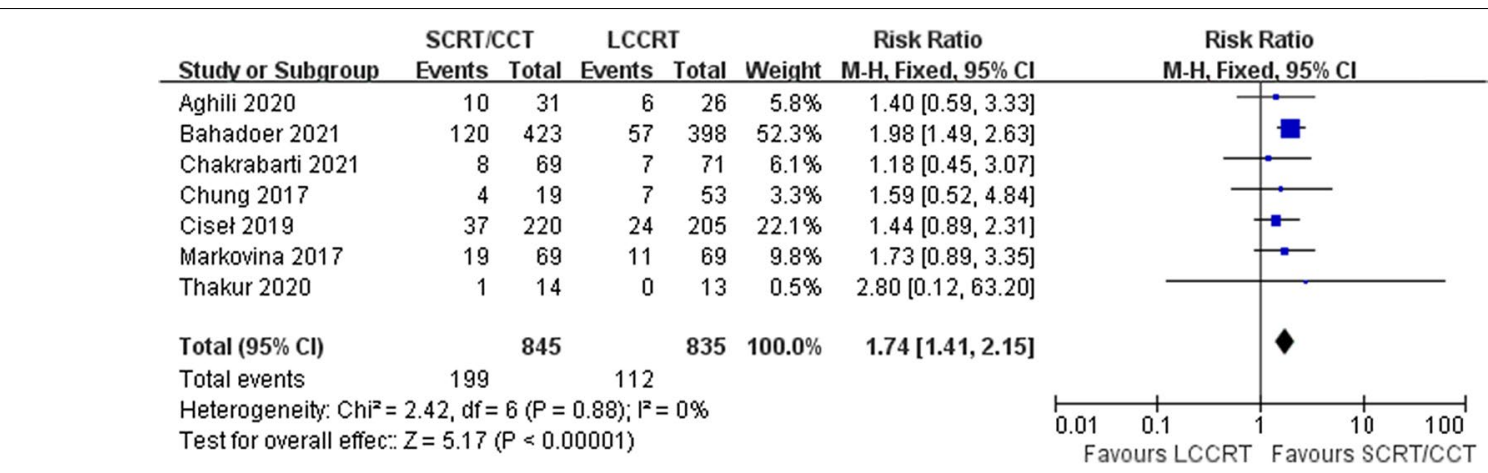

Fig. 2 Forest plot for pathological complete response rate

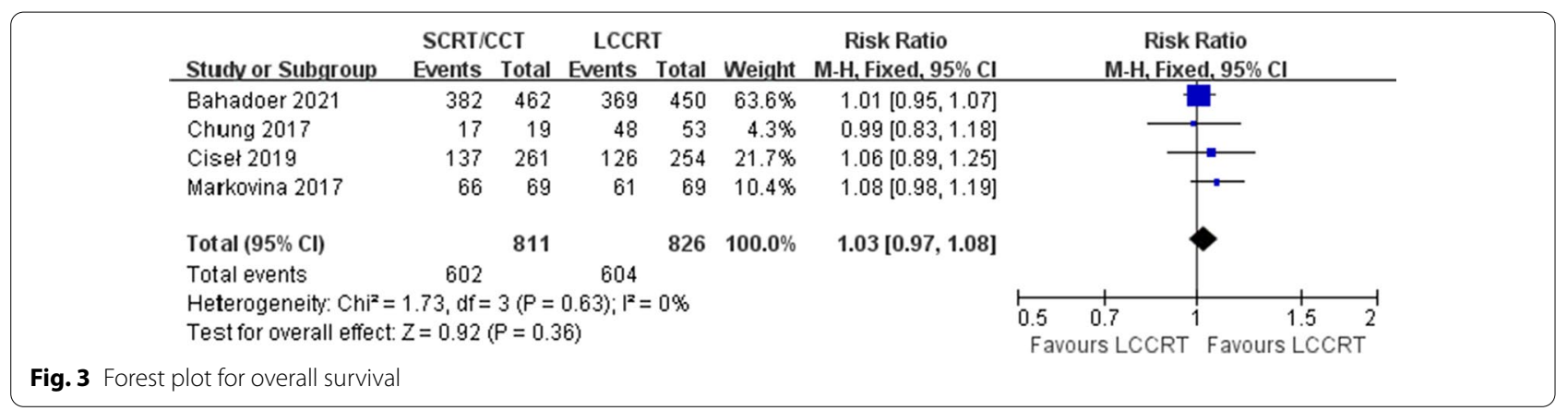

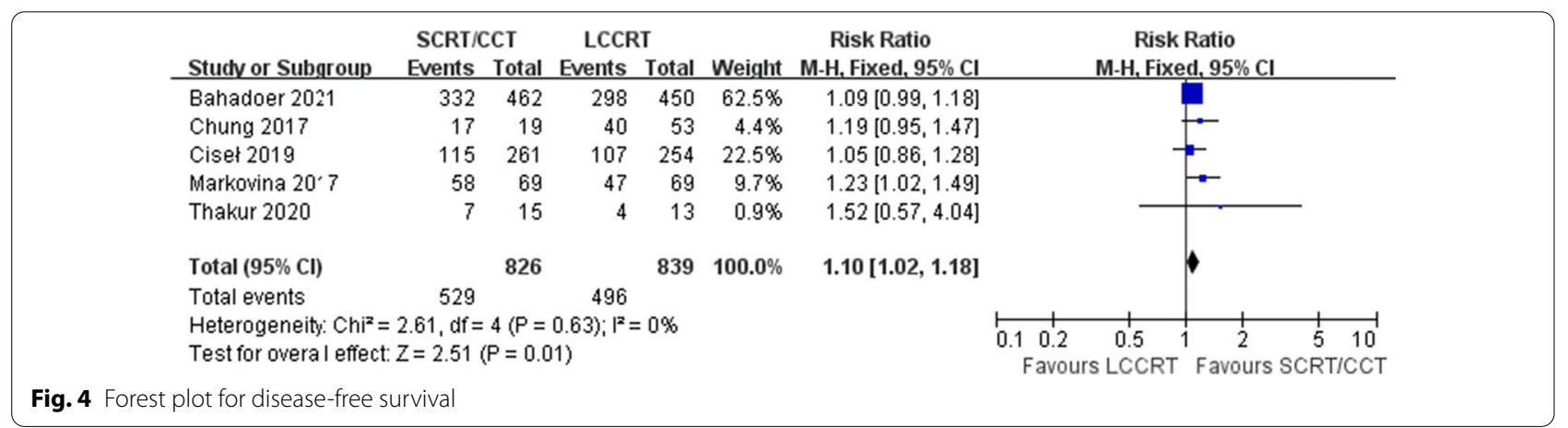

in the SCRT group also likely contributed to improving the tumor response. In a study with four consecutive series of rectal cancer patients receiving $0,2,4$, or 6 cycles of modified FOLFOX6 after identical chemoradiation before surgery, the $\mathrm{pCR}$ rate, compared to that in the chemoradiation alone group, increased by approximately $20 \%$ and was as high as $38 \%$ in the group delivering six cycles of chemotherapy [24]. Obtaining a pCR, referred to as the eradication of all cancer cells, after preoperative treatment is, to some extent, considered synonymous with a cure. Patients with pCR have been associated with improved survival outcomes, as demonstrated by several pooled studies [25-27]. Additionally, patients with a clinical complete response could follow a watch-and-wait strategy, which is increasingly being used as an alternative to major surgery [28].

DFS is largely influenced by local recurrence and systemic relapse. In our meta-analysis, similar events of LC were observed between the two groups. The occurrence of DM, however, was indeed less common in the SCRT/ CCT group, which resulted in better DFS. The differences in DM between the two groups were not statistically significant, as we adopted a random-effect model due to obvious heterogeneity. If a fixed-effect model was applied, the difference would become significant. A meta-analysis suggested that the addition of oxaliplatin 
to 5FU-based chemoradiotherapy resulted in an increase in $\mathrm{pCR}$ and fewer perioperative metastases [29]. In our meta-analysis, almost all patients in the SCRT/CCT group received preoperative chemotherapy containing oxaliplatin. Nevertheless, the reduction in distant metastasis, we suggested, should be attributed to systemic chemotherapy rather than oxaliplatin alone. Improved DFS by consolidation chemotherapy confirmed by a multicenter phase II trial, which evaluated the survival results of LARC patients receiving different cycles of mFOLFOX during the period between chemoradiation and surgery [30]. Compared with that in patients who received only chemoradiation, DFS was better in patients who received additional preoperative chemotherapy. Systemic chemotherapy was also administered in the LCCRT group, but it usually began 6-8 weeks after surgery, which is much later than preoperative chemotherapy. In addition, the rate of adherence to adjuvant chemotherapy is unsatisfactory, as demonstrated by the largest adjuvant trial for LARC (EORTC 22921 study) and one of the included RCTs in which the compliance rate was less than $50 \%$ $[14,31]$. Suboptimal compliance and delays in initiating treatment could possibly diminish the effect of eradicating potential micrometastasis. On the other hand, patients with a better physical condition before surgery were more willing to undergo systemic chemotherapy [14]. With earlier and neoadjuvant delivery of consolidation chemotherapy, SCRT/CCT improved DFS by reducing distant relapse to some extent without compromising local control.

Due to obvious heterogeneity, we adopted a randomeffect model when evaluating acute toxicity and observed no statistical differences between groups. If we employed a fixed-effect model, the SRCT/CCT group was inferior to LCCRT groups. The addition of chemotherapy to preoperative treatment possibly resulted in higher toxicity, which was in line with the conclusion from a phase II trial that randomized patients to chemoradiotherapy and surgery with or without FOLFOX induction therapy [32]. Despite considerable acute toxicity during preoperative therapy in the SCRT/CCT group, there were no significant differences noted in the surgical procedures performed or postoperative complications between the two treatment arms. The late complications in the SCRT/ $\mathrm{CCT}$ group, in our pooled analysis, were inferior with borderline significance $(\mathrm{P}=0.05)$. A long-term follow-up study also showed that patients receiving SCRT, compared to nonirradiated patients, had more postoperative hospitalization due to bowel obstructions and other gastrointestinal complications [33]. It is difficult to indicate that SCRT results in long-term morbidity, as late toxicity is less studied in conventional CRT. To the best of our knowledge, a few randomized trials have demonstrated comparable incidents of late complications between the two treatments during the $3-5$ years follow-up period [1, 34].

The addition of consolidation or induction chemotherapy to concomitant neoadjuvant chemoradiation is both safe and effective, as suggested by some small Phase II studies $[35,36]$. Such neoadjuvant therapy strategy, compared to LCCRT, not only increased pCR rate, but also improved survival rate $[37,38]$, which were similar to our results. It is hard to draw a conclusion whether the addition of neoadjuvant chemotherapy to SCRT or LCCRT is better, as less studies are implemented, making a direct comparison difficult. One thing that is certain is that the delivery of five radiotherapy fractions instead of 25 or 28 fractions not only made short-course radiotherapy cheaper and more convenient than conventional concomitant chemoradiation but also decreased the number of treatment days spent in the medical center, especially in the context of the COVID-19 pandemic. This reduction in time spent in the hospital minimized the risk of COVID-19 infection in these susceptible patients [39].

One of the major limitations of this meta-analysis was that 7 included studies contained 3 non-RCTs. Nonetheless, all included studies were of high quality in accordance with the Newcastle-Ottawa criteria or the Cochrane Collaboration's risk for bias assessment tool. In addition, survival events were calculated at fixed time points as the cumulative survival rate were available only in three studies (two RCTs and one prospective studies). The pooled HR for DFS, again favoring SCRT/CCT, was 0.83 [95\% CI $(0.70,0.97), \mathrm{P}=0.02 ; \mathrm{I}^{2}=38 \%$, fixed model; Additional file 1: Fig. 13], while those for OS was similar between two groups $[\mathrm{HR}=0.90,95 \% \mathrm{CI}(0.74,1.09)$, $\mathrm{P}=0.27 ; \mathrm{I}^{2}=0 \%$, fixed model; Additional file 1: Fig. 14]. Last but not least, the cycles of consolidation chemotherapy varied widely in the SCRT/CCT groups, and the optimal regimen for consolidation chemotherapy is still uncertain.

\section{Conclusion}

In summary, with similar OS, surgical procedures and toxicity but improved DFS and pathological outcomes, SCRT/CCT is a rational alternative neoadjuvant treatment for locally advanced rectal cancer, especially in the context of the COVID-19 pandemic.

\section{Abbreviations}

LARC: Locally advanced rectal cancer; CRT: Conventionally chemoradiotherapy; SCRT: Short-course radiotherapy; pCR: Pathologic complete response; SCRT/CCT: Short-course radiotherapy followed by consolidation chemotherapy; LCCRT: Conventional long-course chemoradiotherapy; ypTNM: Adjuvant therapy pathologic stage; LR: Local recurrence; DM: Distant metastasis; OS: Overall survival rate; DFS: Disease free survival rate; MESH: Medical Subject 
Heading; RCTs:: Randomized controlled trials; RR: Risk ratio; Cl: Confidence intervals.

\section{Supplementary Information}

The online version contains supplementary material available at https://doi. org/10.1186/s13014-021-01974-4.

Additional file 1. Fig. 1: Risk of bias graph. Fig. 2: Risk of bias summary. Table 1: Scores of 4 Cohort Studies Using Newcastle-Ottawa Criteria. Table 2: Patients characteristics of included studies. Fig. 3: Forest plot for downstaging rate. Fig. 4: Forest plot for adjuvant therapy pathologic tumor stage 3-4. Fig. 5: Forest plot for adjuvant therapy pathologic lymph node positive. Fig. 6: Forest plot for local recurrence. Fig. 7: Forest plot for distant metastasis. Fig. 8: Forest plot for acute toxicity. Fig. 9: Forest plot for postoperative complications. Fig. 10: Forest plot for late complications. Fig. 11: Forest plot for R0 resection rate. Fig. 12: Forest plot for sphincter preservation rate. Fig. 13: Forest plot for disease-free survival. Fig. 14: Forest plot for overall survival.

\section{Acknowledgements}

Not applicable.

\section{Authors' contributions}

HW, CF, and CF made substantial contributions to literature search, study design and data collection. $\mathrm{LH}$ and $\mathrm{YL}$ participated in data analysis and data interpretation. HW, CF, and CF finished the first draft and $Y L$ and $Z Z$ participated in revising it critically for important intellectual content; $Y L$ and $Z Z$ gave final approval of the version to be published. HW, CF, and CF contributed equally to this work. All authors read and approved the final manuscript.

\section{Funding}

The study was not funded.

\section{Availability of data and materials}

All data generated or analyzed during this study are included in this manuscript.

\section{Declarations}

Ethics approval and consent to participate

All analyses were based on previously published studies, and hence no ethical approval and patient consent were required.

\section{Consent for publication}

Not applicable.

\section{Competing interests}

The authors declare that they have no competing interests.

\section{Author details}

${ }^{1}$ Laboratory of Digestive Surgery, State Key Laboratory of Biotherapy and Cancer Center, Sichuan University, Chengdu 610041, China. ${ }^{2}$ Department of Gastrointestinal Surgery, West China Hospital, West China School of Medicine, Sichuan University, No. 37, Guo Xue Xiang, Chengdu 610041, China. ${ }^{3}$ Department of Pediatric Surgery, West China Hospital, West China School of Medicine, Sichuan University, Chengdu 610041, China. ${ }^{4}$ Department of Gastrointestinal Surgery and Breast and Thyroid Surgery, Minimally Invasive Surgery, West China School of Public Health, Sichuan University, Chengdu 610041, China. ${ }^{5}$ West China Fourth Hospital, Sichuan University, Chengdu 610041, China. ${ }^{6}$ Department of Oncology, Linköping University, 58183 Linköping, Sweden. ${ }^{7}$ Department of Biomedical and Clinical Sciences, Linköping University, 58183 Linköping, Sweden.

Received: 1 September 2021 Accepted: 21 December 2021

Published online: 24 January 2022

\section{References}

1. Bujko K, Nowacki MP, Nasierowska-Guttmejer A, Michalski W, Bebenek M, Kryj M. Long-term results of a randomized trial comparing preoperative short-course radiotherapy with preoperative conventionally fractionated chemoradiation for rectal cancer. Br J Surg. 2006:93:1215-23.

2. Rödel C, Trojan J, Bechstein WO, Woeste G. Neoadjuvant short- or longterm radio(chemo)therapy for rectal cancer: how and who should be treated? Dig Dis (Basel, Switzerland). 2012;30(Suppl 2):102-8.

3. Erlandsson J, Holm T, Pettersson D, Berglund Å, Cedermark B, Radu C, et al. Optimal fractionation of preoperative radiotherapy and timing to surgery for rectal cancer (Stockholm III): a multicentre, randomised, nonblinded, phase 3, non-inferiority trial. Lancet Oncol. 2017;18:336-46.

4. Ngan SY, Burmeister B, Fisher RJ, Solomon M, Goldstein D, Joseph D, et al. Randomized trial of short-course radiotherapy versus long-course chemoradiation comparing rates of local recurrence in patients with T3 rectal cancer: trans-Tasman Radiation Oncology Group trial 01.04. J Clin Oncol. 2012;30:3827-33.

5. Eitta MA, El-Wahidi GF, Fouda MA, El-Hak NG, Abo El-Naga EM Preoperative radiotherapy in resectable rectal cancer: a prospective randomized study of two different approaches. J Egypt Natl Canc Inst. 2010;22:155-64.

6. Pach R, Kulig J, Richter P, Gach T, Szura M, Kowalska T. Randomized clinical trial on preoperative radiotherapy $25 \mathrm{~Gy}$ in rectal cancer-treatment results at 5-year follow-up. Langenbecks Arch Surg. 2012;397:801-7.

7. Erlandsson J, Lörinc E, Ahlberg M, Pettersson D, Holm T, Glimelius B, et al. Tumour regression after radiotherapy for rectal cancer-results from the randomised Stockholm III trial. Radiother Oncol. 2019;135:178-86.

8. Bujko K, Partycki M, Pietrzak L. Neoadjuvant radiotherapy $(5 \times 5$ Gy): immediate versus delayed surgery. Recent results in cancer research. Fortschritte der Krebsforschung Progres dans les recherches sur le cancer. 2014:203:171-87.

9. Bahadoer RR, Dijkstra EA, van Etten B, Marijnen CAM, Putter H, Kranenbarg EM, et al. Short-course radiotherapy followed by chemotherapy before total mesorectal excision (TME) versus preoperative chemoradiotherapy, TME, and optional adjuvant chemotherapy in locally advanced rectal cancer (RAPIDO): a randomised, open-label, phase 3 trial. Lancet Oncol. 2021;22:29-42.

10. Bosset JF, Collette L, Calais G, Mineur L, Maingon P, Radosevic-Jelic L, et al. Chemotherapy with preoperative radiotherapy in rectal cancer. N Engl J Med. 2006;355:1114-23.

11. Breugom AJ, van Gijn W, Muller EW, Berglund $\AA$, van den Broek CBM, Fokstuen T, et al. Adjuvant chemotherapy for rectal cancer patients treated with preoperative (chemo)radiotherapy and total mesorectal excision: a Dutch Colorectal Cancer Group (DCCG) randomized phase III trial. Ann Oncol. 2015;26:696-701.

12. van DijkTH, Tamas K, Beukema JC, Beets GL, Gelderblom AJ, de Jong $K P$, et al. Evaluation of short-course radiotherapy followed by neoadjuvant bevacizumab, capecitabine, and oxaliplatin and subsequent radical surgical treatment in primary stage IV rectal cancer. Ann Oncol. 2013;24:1762-9.

13. Jia AY, Narang A, Safar B, Zaheer A, Murphy A, Azad NS, et al. Sequential short-course radiation therapy and chemotherapy in the neoadjuvant treatment of rectal adenocarcinoma. Radiat Oncol (London, England). 2019;14:147.

14. van der Valk MJM, Marijnen CAM, van Etten B, Dijkstra EA, Hilling DE, Kranenbarg EM, et al. Compliance and tolerability of short-course radiotherapy followed by preoperative chemotherapy and surgery for highrisk rectal cancer-results of the international randomized RAPIDO-trial. Radiother Oncol. 2020;147:75-83.

15. Huang L, Yin Y, Yang L, Wang C, Li Y, Zhou Z. Comparison of antibiotic therapy and appendectomy for acute uncomplicated appendicitis in children: a meta-analysis. JAMA Pediatr. 2017;171:426-34.

16. Stang A. Critical evaluation of the Newcastle-Ottawa scale for the assessment of the quality of nonrandomized studies in meta-analyses. Eur J Epidemiol. 2010;25:603-5.

17. Ciseł B, Pietrzak L, Michalski W, Wyrwicz L, Rutkowski A, Kosakowska E, et al. Long-course preoperative chemoradiation versus $5 \times 5$ Gy and consolidation chemotherapy for clinical T4 and fixed clinical T3 rectal cancer: long-term results of the randomized Polish II study. Ann Oncol. 2019;30:1298-303. 
18. Chakrabarti D, Rajan S, Akhtar N, Qayoom S, Gupta S, Verma M, et al. Short-course radiotherapy with consolidation chemotherapy versus conventionally fractionated long-course chemoradiotherapy for locally advanced rectal cancer: randomized clinical trial. Br J Surg. 2021. https:// doi.org/10.1093/bjs/znab020.

19. Aghili M, Khalili N, Khalili N, Babaei M, Farhan F, Haddad P, et al. Shortcourse versus long-course neoadjuvant chemoradiotherapy in patients with rectal cancer: preliminary results of a randomized controlled trial. Radiat Oncol J. 2020:38:119-28.

20. Markovina S, Youssef F, Roy A, Aggarwal S, Khwaja S, DeWees T, et al. Improved metastasis- and disease-free survival with preoperative sequential short-course radiation therapy and FOLFOX chemotherapy for rectal cancer compared with neoadjuvant long-course chemoradiotherapy: results of a matched pair analysis. Int J Radiat Oncol Biol Phys. 2017;99:417-26.

21. Chung MJ, Kim DW, Chung WK, Lee SH, Jeong SK, Hwang JK, et al. Preoperative short- vs. long-course chemoradiotherapy with delayed surgery for locally advanced rectal cancer. Oncotarget. 2017;8:60479-86.

22. Thakur N, Seam RK, Gupta MK, Gupta M, Fotedar V, Vats S, et al. A prospective observational study comparing long-course conventional neoadjuvant chemoradiotherapy with short-course radiotherapy followed by consolidation chemotherapy with delayed surgery in locally advanced rectal cancer. South Asian J Cancer. 2020;9:80-5.

23. Francois Y, Nemoz CJ, Baulieux J, Vignal J, Grandjean JP, Partensky C, et al. Influence of the interval between preoperative radiation therapy and surgery on downstaging and on the rate of sphincter-sparing surgery for rectal cancer: the Lyon R90-01 randomized trial. J Clin Oncol. 1999;17:2396

24. Garcia-Aguilar J, Chow OS, Smith DD, Marcet JE, Cataldo PA, Varma MG, et al. Effect of adding mFOLFOX6 after neoadjuvant chemoradiation in locally advanced rectal cancer: a multicentre, phase 2 trial. Lancet Oncol. 2015;16:957-66.

25. Maas M, Nelemans PJ, Valentini V, Das P, Rödel C, Kuo LJ, et al. Long-term outcome in patients with a pathological complete response after chemoradiation for rectal cancer: a pooled analysis of individual patient data. Lancet Oncol. 2010;11:835-44.

26. Martin ST, Heneghan HM, Winter DC. Systematic review and meta-analysis of outcomes following pathological complete response to neoadjuvant chemoradiotherapy for rectal cancer. Br J Surg. 2012;99:918-28.

27. Zorcolo L, Rosman AS, Restivo A, Pisano M, Nigri GR, Fancellu A, et al. Complete pathologic response after combined modality treatment for rectal cancer and long-term survival: a meta-analysis. Ann Surg Oncol. 2012;19:2822-32.

28. van der Valk MJM, Hilling DE, Bastiaannet E, Meershoek-Klein Kranenbarg E, Beets GL, Figueiredo NL, et al. Long-term outcomes of clinical complete responders after neoadjuvant treatment for rectal cancer in the International Watch \& Wait Database (IWWD): an international multicentre registry study. Lancet (London, England). 2018;391:2537-45.

29. An X, Lin X, Wang FH, Goodman K, Cai PQ, Kong LH, et al. Short term results of neoadjuvant chemoradiotherapy with fluoropyrimidine alone or in combination with oxaliplatin in locally advanced rectal cancer: a meta analysis. Eur J Cancer (Oxford, England: 1990). 2013;49:843-51.

30. Marco MR, Zhou L, Patil S, Marcet JE, Varma MG, Oommen S, et al. Consolidation mFOLFOX6 chemotherapy after chemoradiotherapy improves survival in patients with locally advanced rectal cancer: final results of a Multicenter Phase II Trial. Dis Colon Rectum. 2018;61:1146-55.

31. Bosset JF, Calais G, Mineur L, Maingon P, Stojanovic-Rundic S, Bensadoun RJ, et al. Fluorouracil-based adjuvant chemotherapy after preoperative chemoradiotherapy in rectal cancer: long-term results of the EORTC 22921 randomised study. Lancet Oncol. 2014;15:184-90.

32. Maréchal $R$, Vos B, Polus M, Delaunoit T, Peeters M, Demetter $P$, et al. Short course chemotherapy followed by concomitant chemoradiotherapy and surgery in locally advanced rectal cancer: a randomized multicentric phase II study. Ann Oncol. 2012;23:1525-30.

33. Birgisson H, Påhlman L, Gunnarsson U, Glimelius B. Adverse effects of preoperative radiation therapy for rectal cancer: long-term follow-up of the Swedish Rectal Cancer Trial. J Clin Oncol. 2005;23:8697-705.

34. Ansari N, Solomon MJ, Fisher RJ, Mackay J, Burmeister B, Ackland S, et al. Acute adverse events and postoperative complications in a randomized trial of preoperative short-course radiotherapy versus long-course chemoradiotherapy for T3 adenocarcinoma of the rectum: trans-Tasman Radiation Oncology Group Trial (TROG 0104). Ann Surg. 2017;265:882-8.

35. Chua YJ, Barbachano Y, Cunningham D, Oates JR, Brown G, Wotherspoon A, et al. Neoadjuvant capecitabine and oxaliplatin before chemoradiotherapy and total mesorectal excision in MRI-defined poor-risk rectal cancer: a phase 2 trial. Lancet Oncol. 2010;11:241-8.

36. Schou JV, Larsen FO, Rasch L, Linnemann D, Langhoff J, Høgdall E, et al. Induction chemotherapy with capecitabine and oxaliplatin followed by chemoradiotherapy before total mesorectal excision in patients with locally advanced rectal cancer. Ann Oncol. 2012;23:2627-33.

37. Petrelli F, Trevisan F, Cabiddu M, Sgroi G, Bruschieri L, Rausa E, et al. Total neoadjuvant therapy in rectal cancer: a systematic review and metaanalysis of treatment outcomes. Ann Surg. 2020;271:440-8.

38. Riesco-Martinez MC, Fernandez-Martos C, Gravalos-Castro C, EspinosaOlarte P, La Salvia A, Robles-Diaz L, et al. Impact of total neoadjuvant therapy vs. standard chemoradiotherapy in locally advanced rectal cancer: a systematic review and meta-analysis of randomized trials. Cancers. 2020;12:3655.

39. Marijnen CAM, Peters FP, Rödel C, Bujko K, Haustermans K, Fokas E, et al. International expert consensus statement regarding radiotherapy treatment options for rectal cancer during the COVID 19 pandemic. Radiother Oncol. 2020;148:213-5.

\section{Publisher's Note}

Springer Nature remains neutral with regard to jurisdictional claims in published maps and institutional affiliations.

Ready to submit your research? Choose BMC and benefit from

- fast, convenient online submission

- thorough peer review by experienced researchers in your field

- rapid publication on acceptance

- support for research data, including large and complex data types

- gold Open Access which fosters wider collaboration and increased citations

- maximum visibility for your research: over 100M website views per year

At BMC, research is always in progress.

Learn more biomedcentral.com/submissions 